\title{
LA LONGUE MARCHE DES FEMMES HAÏTIENNES: \\ DE LA SOEUR À LA MÈRE
}

HAITIAN WOMEN'S LONG MARCH: FROM SISTER TO MOTHER

Maximilien Laroche

Université Laval

Québec, Canada

\begin{abstract}
Since the first novel published in 1859, haitian novelists have traditionally described the necessity of democratic change and modernization of haitian society as a fight of brothers. In this fight, sisters have intervene first as auxiliary but more and more as leaders. According to the evolution of this tradition it appears that reconciliation of brothers will not be sufficient if there is no co-operation between brothers and sisters in their fight.
\end{abstract}

Keywords: Haitian literature; Tradition; Cooperation; Literary generations; Women fight.

\section{Résumé}

Dès le premier roman publié en 1959 , les romanciers haïtiens ont traditionnellement représenté le besoin de démocratiser et de moderniser la société haïtienne par l'image d'une lutte de frères ennemis. Les sœurs ont toujours participé à cette lutte. D'abord en tant qu'auxiliaires et de plus en plus comme leaders. Cette tradition qui évolue démontre qu'il ne suffira pas que les frères se réconcilient mais qu'il faudra que frères et sœurs coopèrent dans leur lutte.

Mots-clés: Littérature haïtienne; Tradition; Coopération; Générations littéraires; Lutte des femmes.

\section{Resumo}

Desde o primeiro romance publicado em 1959, os romancistas haitianos representaram tradicionalmente a necessidade de democratizar e de modernizar a sociedade haitiana pela imagem de uma luta de irmãos inimigos. As irmás sempre participaram desta luta. Primeiramente enquanto auxiliares e cada vez mais como líderes. Esta tradição que evolui demonstra que não bastará que os irmãos se reconciliem, mas que será preciso que os irmãos e as irmãs cooperem em sua luta.

Palavras-chave: Literatura haitiana, Tradição, Cooperação, Geraçôes literárias, Luta das mulheres

Dans les premières pages de son ouvrage, Formação da literatura brasileira, au chapitre intitulé : "Literatura como sistema ", Antonio Candido donne cette définition de la tradition littéraire :

[...] convém principiar distinguindo manifestaçóes literárias, de literatura propriamente dita, considerada aqui un sistema de obras ligadas por denominadores 
comuns [...] Estes denominadores são, além das características internas [...] certos elementos de natureza social e psíquica embora literariamente organizados, que se manifestam historicamente et fazem da literatura aspecto orgânico da civilizaçăo [...] se distinguem a existência de um conjunto de produtores literários...um conjunto de receptores [...] um mecanismo transmissor [...] O conjunto dos três elementos dá lugar a um tipo de communicação interhumana, a literatura que aparece sob este ângulo como sistema simbólico (1993: 23)

Et le critique brésilien, un peu plus loin, ajoute:

[...] Quando a atividade dos escritores de um dado período se integra em tal sistema, ocorre outro elemento decisivo: a formaçáo da continuidade literária [...] que assegura no tempo o movimento conjunto, definindo os lineamentos de um todo. É uma tradição, no sentido completo do termo, isto é, transmissão de algo entre os homens, e o conjunto de elementos transmitidos, formando padrôes que se impôem ao pensamento ou ao comportamento, e aos quais somos obrigados a nos referir, para aceitar ou rejeitar. Sem esta tradição não há literatura, como fenômeno de civilizaçấo. (1993: 24)

Je crois qu'il s'agit là d'une définition taillée sur mesure, dont je vais m’inspirer pour retracer, de la sœur à la mère, l'évolution d'une représentation de la lutte des femmes dans le roman haïtien.

\section{Polysémie du terme manman}

Même l'Haïtien qui utilise avec la plus grande pertinence les mots de sa langue natale, le créole haïtien, le fait le plus souvent en ignorant l'ensemble des performances stylistiques de sa langue, faute de l'avoir étudiée. Il pourrait, par exemple, s'écrier, devant un spectacle qui s'offre à lui : «Kou Manman lanman!» (Comme manman lanman!) et croire qu'il ne fait que se servir de mots par simple allitération. Il ignorerait que « manman lanman » est le nom d'une plante : physalis, à propos de laquelle le dictionnaire Robert nous dit ceci : « Plante vivace (solanacées) appelée aussi amour en cage, dont le calice renflé contenant le fruit à maturité est très décoratif. » L'exclamation de ce locuteur était en fait une métaphore célébrant la merveille de la mère portant en elle la vie nouvelle, comme la plante évoquée. Ce n'était pas une simple musique de mots sans liens logiques. «Kou manman lan » serait ainsi un équivalent de l'admiratif : "Mama mia!»

En haïtien, c'est-à-dire dans la langue créole des Haïtiens, « Manman » correspond à « Mamman » en français. Manman et, sous sa forme abrégée : " Man », renvoient généralement à « Manman pitit » (la mère d'un enfant) et naturellement il sert à traduire toute la tendresse ou la vénération d'un enfant pour sa mère. Et c'est ce que traduit ce vers d'une chanson où l'on dit tautologiquement : «Manman, se manman!» (une mère, c'est une mère). 
D'ailleurs il n'est pas rare d'entendre quelqu'un, s'adressant à une vieille dame dont il ignore le nom, l'appeler, par politesse : « la mère ». Mais plus couramment on se sert de manman pour s'adresser à sa propre mère ou pour parler de la mère de quelqu'un.

Le mot manman peut désigner aussi la femme aimée, celle, en particulier, qui nous fait souffrir, comme on le dit dans cette chanson :

Ay manman Nanòt! (Oh, Nanòt, chérie !)

Pouki sa ou ap fe m soufri ? (Pourquoi me fais-tu souffrir ?) ;

Cette souffrance peut n'être souvent que la plainte de celui qui s'étonne hypocritement d'être repoussé :

Eske se paske m pap travay (Est-ce parce que je ne travaille pas,)

$\mathrm{Ou}$ ap fè $\mathrm{m}$ pase yon tray? (que tu me fais des misères ?).

Alors le mot manman, synonyme de l'indulgence sans limite, autorise alors l'amoureux à quémander les faveurs de sa belle, comme il le fait dans cette autre chanson :

Ti manman fè kichoy pou mwen (Petite mère, fais-moi plaisir !)

Pa kite $\mathrm{m}$ ale ! (Ne me renvoie pas!)

Le mot Man, abrégé de mamman, peut s'associer aussi bien avec le nom de la personne désignée (Man Cia) qu'avec celui du conjoint de cette personne, comme dans Man Dodo (la femme de Dodo) qui prend le sens de "Manman pitit Dodo" (la mère des enfants de Dodo).

Sur le registre des sentiments, le mot Manman ou son diminutif Man peut donc traduire toute une gamme de sentiments et de situations. Et c'est ainsi qu'il élargit son sens pour désigner non seulement des personnes mais même des objets comme dans manman kay (la grande maison), manman lajan an (le gros du trésor) ou encore Man Pinba, le nom donné aux canons de gros calibre placés dans la Citadelle Laferierre pour défendre le pays dans le cas d'un retour des Français.

Il y a donc une sorte de réversibilité du mot "Manman " ou de son équivalent «Man » qui lui permet, tantôt nom, tantôt épithète, de passer de l'amour à la guerre ou si l'on préfere du positif au négatif. Il peut désigner alors tout ce qui est imposant, étonnant ou même menaçant. Le terme Manman renvoie alors à tout ce qui dépasse les proportions ordinaires des choses, ce qui est impressionnant, terrifiant à la limite. On parle alors de " Manman loup-garou "pour désigner les sorcières qui rodent la nuit ou de «San manman " pour parler de gens sans foi ni loi. Le terme Manman peut aussi servir pour insulter. Et si ailleurs, on le fait elliptiquement en disant : « Eh! Ta mère ! ", en haïtien, on le fait plus crûment en lançant : « Kou langèt manman ou " (Comme le clitoris te ta mère!). 
C'est dans cette perspective d'un élargissement constant du sens du mot Manman qu'on arrive à l'employer pour parler du pays, la mère patrie en somme, avec toutes les nuances d'affection, de vénération, de nostalgie, mais aussi de culpabilité ou même d'espoir désespéré, si l'on peut ainsi parler. À preuve, ces vers d'une chanson faisant la louange d'Haïti et qui se terminent par ce souhait :

Ayiti, Ayiti, (Haïti ! Haïti !)

Ayiti, manman cheri, (Haïti, mère chérie)

Se nan bra ou pou m mouri. (Je veux mourir dans tes bras.)

Est-ce le rêve de retrouver le confort et la paix du fotus qui n'a pas encore affronté le monde tel qu'il est ou celui de renaître à une vie nouvelle, comme semblait le souhaiter le roi Christophe dont l'emblème était le phénix, l'oiseau qui renait de ses cendres? Cette image de la mort dans les bras de la mère chérie nous porte à croire que dans ce cas, le rêve ultime du poète est double : d'abord mettre fin aux malheurs présents et ensuite recommencer la vie avec un avenir plus clément. Se reforger en somme une nouvelle histoire.

Mais dans ce rêve de réincarnation, s'insinue souvent le sentiment de culpabilité de ne pas avoir suffisamment témoigné de tendresse à cette mère qui nous a donné le jour ou même le regret de s'être montré ingrat à son égard. Il y a ainsi pas mal de chansons qui dénoncent le fils ingrat qui s'est élevé dans l'échelle sociale grâce aux sacrifices d'une mère qu'à présent il méprise et rejette.

Ces diverses attitudes à l'égard de la mère-patrie s'insèrent dans une vision extrêmement large de la famille. Un dicton populaire ne dit-il pas : « Wzazinay, se fanmi. (Les voisins sont des parents) ». Le parent ce n'est donc pas seulement le membre de la famille nucléaire mais le voisin, celui qui habite la même rue, le même quartier, la même ville, le même pays ou encore ces voisins qui habitent de l'autre côté de la frontière, la même île, le même continent et à la limite, la même planète terre. Un passage de Gouverneurs de la rosée illustre bien cette vision de l'autre comme moi, à l'opposé du fameux : " Je est un autre. » :

Et moi, aussi, cria le Simidor. Il venait d'avaler coup sur coup deux verres de clairin. J'ai traversé plusieurs fois la frontière : ces Dominicains-là, ce sont des gens comme nous-mêmes, sauf qu' ils ont une couleur plus rouge que les nègres d'Haïti, et leurs femmes sont des mulâtresses à grande crinière. J'ai connu une de ces bougresses, elle était bien grasse, pour dire la vérité. Antonio, qu'elle m'appelait, voilà comment elle m'appelait. Eh bien, question de comparaison avec les femmes d'icitte, rien ne lui manquait. Elle avait de tout et de bonne qualité. Je pourrais faire un serment, mais Destine me criera après. Destine chérie, ce n'est pas la langue qui compte, non, c'est le reste, tu peux me croire.» (ROUMAIN, 2003: 287)

La vision de tous ces voisins qui seraient de la même famille que nous n'est malheureusement pas toujours la règle. L'histoire d'Haïti serait même 
plutôt le témoignage du contraire. Aussi bien par ce qui se passe entre les Haïtiens eux-mêmes qu'entre le reste du monde et Haïti. Les Haïtiens sont divisés entre eux, en même temps qu'ils se sentent exclus du reste du monde. Et c'est dans cette double perspective historique que s'insère, dans le roman haïtien, la vision d'Haïti comme figure de la famille divisée par la lutte des frères.

\section{De Stella à Bicentenaire}

Le premier roman haïtien, Stella d'Emeric Bergeaud, fut publié en 1859. Bicentenaire, un roman de Lyonel Trouillot, date de 2004, l'année même du deux centième anniversaire de l'indépendance haïtienne.

On pourrait dire de ces deux romans qu'ils sont des fictions sur l'histoire politique d'Haïti d'hier et d'aujourd'hui. Le premier roman raconte l'histoire de la conquête de l'indépendance en 1804 et l'origine des guerres civiles qui en résultèrent. Le deuxième évoque les tribulations actuelles de ceux qui voudraient voir le pays se libérer du despotisme et de l'incurie de ses dirigeants.

Là où on peut faire un parallèle entre ces deux récits, c'est qu'ils utilisent un même schéma narratif. Il s'agit, dans Stella, de la lutte de deux frères, Romulus et Rémus, qui d'abord se battent contre le colon français, père de l'un d'eux, et qui a maltraité leur mère, Marie l'Africaine. Puis, une fois cette première guerre terminée, Stella fait le récit de la guerre fratricide à laquelle ces deux frères ont commencé à se livrer pour la conquête du pouvoir. Dans Bicentenaire, il y a aussi deux frères : l'étudiant et son frère cadet. Les deux ont laissé leur mère, Ernestine Saint-Hilaire, aveugle et abandonnée, dans une campagne éloignée, pour se rendre dans la capitale. Et là, l'aîné milite contre le gouvernement en place tandis que son jeune frère soutient ce gouvernement.

Or si Marie l'Africaine est morte, c'est tout comme pour Ernestine SaintHilaire, qui vit aveugle et isolée dans une région pauvre du pays. Les deux frères, dans ces deux romans, auraient pu avoir une amie, une sœur, qui les aurait dissuadés de se battre et aurait ramené la paix entre eux. Mais l'ange de la liberté, Stella, qui avait aidé Romulus et Rémus, dans leur lutte contre le colon esclavagiste, s'est envolée et a quitté le pays après la proclamation de l'indépendance. Et l'étudiant de Bicentenaire à qui une journaliste étrangère servait de muse, a vu celle-ci, elle aussi, quitter le pays.

À deux siècles de distance, la situation demeure donc bloquée dans ce conflit familial qui nous est représenté, dans un premier cas, par une allégorie, puisque Stella est cet ange venu de France et qui y retourne, après la conquête de l'indépendance en 1804. Et dans le deuxième cas, on s'aperçoit que, dans cette métaphore de la situation politique actuelle qu'est Bicentenaire la journaliste étrangère tout comme Stella, aurait pu être de bon conseil pour les deux frères, si elle n'avait, elle aussi, quitté Haïti. 
Cette situation qui perdure, met donc en scène, dans un rapport conflictuel, les membres d'une même famille. Entendez par là des personnages qui partagent le même lien de sang, de sentiment ou d'idéologie et dont la représentation s'échelonne dans des œuvres romanesques publiées pendant près de deux siècles, de 1859 à 2004.

\section{Les romanciers de la Ronde}

À la mort du père, les frères se battent d'ordinaire pour sa succession. Et c'est dans ce cas qu'on parle de lutte des frères ennemis. Mais il arrive aussi que le père mort, un sosie lui succède, et c'est là aussi un motif de querelle entre deux frères. Car l'un peut s'accommoder d'un nouveau parâtre avec lequel il pactise alors qu'un autre frère peut vouloir continuer le combat pour le renversement de cette réplique de l'ancien parâtre.

Emeric Bergeaud, dans Stella, en 1859, nous racontait d'abord la lutte de Romulus et de Rémus contre le colon esclavagiste, leur victoire et ensuite le déclenchement de cette guerre pour sa succession qui perdure. Les romanciers de $L a$ Ronde, cette école littéraire qui s'imposa sur la scène littéraire, entre 1900 et 1925, nous décrivent, quant à eux, la situation qui prévalait un siècle plus tard alors que cette indépendance acquise n’avait pas mis fin à la tyrannie de dirigeants comparables aux colonisateurs d'autrefois. La même lutte des frères se poursuivait donc mais cette fois à propos d'un nouveau parâtre presque identique au premier. Et les frères qui s'entredéchiraient pouvaient se répartir en partisans et en adversaires de ce sosie de l'ennemi d'autrefois. La guerre de libération nationale se changeait donc en guerre civile, en lutte d'un clan de la société contre l'oppression d'un autre clan. Le schéma de la lutte des frères ennemis, dans le roman haïtien, dès le début, se définit donc par un affrontement des frères, dans l'ombre d'un père, invisible souvent, et d'une mère parfois absente mais presque toujours en présence d'une sœur.

Car ainsi que nous l'avons signalé, les frères qui avaient été soutenus, dans un premier temps, dans leur lutte contre le parâtre, par un adjuvant féminin, l'Ange de la Liberté, dans Stella d'Emeric Bergeaud, et la journaliste étrangère, dans Bicentenaire de Lyonel Trouillot, ont vu celles-ci s'éloigner de la scène du combat à partir du moment où ils allaient s'affronter dans une lutte fratricide. Or à partir de l'époque de La Ronde, nous assisterons au remplacement des sœurs étrangères par d'autres sœurs plus proches, notamment, comme nous le disions, par des liens de sang, de cœur ou d'idéologie. On peut donc voir dans cette substitution, non pas tant un abandon mais un retrait de ces sœurs étrangères, à partir du moment où les frères passaient de la révolution à la révolte. Elles laissaient aux combattants le soin de régler 
leur lutte intestine, eux-mêmes, avec l'aide de leurs proches. Trois romans de cette époque de La Ronde nous font saisir quelle contribution les femmes ont apportée dans cet affrontement des frères.

Commençons par deux romans de Frédéric Marcelin : Thémistocle Épaminondas Labasterre, publié en 1901, et La vengeance de Mama qui parut en 1902. Dans le premier roman, l'auteur nous raconte l'histoire d'un jeune homme, Thémistocle Épaminondas Labasterre, qui d'abord fit partie du cercle des admirateurs de Télémaque, un politicien qui s'était fait le critique virulent des mauvaises politiques du gouvernement. Mais sitôt nommé ministre, Télémaque changea du tout au tout et se mit à appliquer les mêmes politiques qu'il critiquait auparavant. Cela porta Thémistocle à dénoncer la volte-face de son ancienne idole. Il fut tué par les sbires de Télémaque. Le deuxième roman, La vengeance de Mama, qui fait suite au roman précédent, porte bien son titre. Il nous raconte la vengeance qu'exerça Mama, la fiancée de Thémistocle, contre Télémaque. Elle tendit un guet-apens au ministre félon qu'elle tua pour ensuite se suicider. Ces deux romans de Marcelin mettent donc en scène deux anciens frères d'armes, devenus d'irréductibles ennemis. Nous avons là le schéma d'un conflit familial mettant en présence deux frères en quelque sorte, mais de convictions politiques opposées, dont l'un meurt par la faute de l'autre. Il y a une mère, celle du journaliste tué, qui appelle à la vengeance et la fiancée du défunt qui met ce souhait à exécution et se suicide par après. Nous retrouverons plus tard ce schéma de l'association d'une mère et de sa bru dans d'autres œuvres mais avec des variantes et des significations opposées. En attendant, considérons un autre roman de l'époque de La Ronde où c'est la mère et sa fille de sang qui, cette fois, s'associent.

Fernand Hibbert, un contemporain de Fréderic Marcelin, dans son roman Les Thazar, nous raconte une histoire à caractère plus social que politique. Cilotte, la fille de monsieur et madame Thazar, doit se décider entre deux prétendants à sa main. D'une part, il y a, comme nous dit Pradel Pompilus, : «Lionel Brion, un beau type d'Haïtien cultivé, écrivain et avocat et un jeune Allemand, Schlieden, assez vulgaire, mais bien argenté. Cilotte aime Brion avec passion, moins cependant que la vie brillante dont elle rêve et que l'étranger peut seul lui offrir. » (: 603). Encouragée par sa mère, elle rejette son prétendant haïtien en lui faisant cet aveu : «...J'épouse Schlieden parce que je ne veux pas de la vie petite, mesquine, tracassière des ménages sans fortune...C'est pourquoi je me suis juré que je ne ferais qu'un mariage solide, garanti avec un étranger bien posé. Évidemment tu n'es pas un pauvre diable, mais tu es Haïtien, et ton petit avoir est à la merci du plus infime bouleversement. À ton premier exil, ce sera fait du peu que tu as : or, je ne veux pas souffrir... » (1975: 195). En cela elle était en parfait accord avec sa mère 
qui déclarait férocement à son mari, au cours d'une dispute : «L'argent est tout » Et, comme dit, Pradel Pompilus : «puisque l'argent est tout, on lui sacrifiera tout : amitié, honneur, pudeur même. » (1975: 613). Dans cette situation-ci, une mère ne s'associait pas avec la fiancée de son fils, comme c'était le cas dans La Vengeance de Mama, pour venger ce fils mais pour tuer dans l'œuf les prétentions de celui qui aurait voulu devenir son gendre. Nous demeurons donc dans le même schéma d'un rapport conflictuel de frères, supervisé par la mère et sa fille, et où la fille, cette fois, fait faux bon à celui qui serait le mieux qualifié pour devenir le gendre de sa mère., son frère en quelque sorte. Cilotte Thazar qui craignait tant, en épousant un Haïtien, de se retrouver en exil, dans la misère, avant de suivre son mari en Allemagne, viendra faire cet aveu, à son ancien prétendant qu'elle avait voulu revoir : «C'est triste, la vie...» (: 222).

Il nous reste à examiner une variante encore plus étonnante de ce rapport de forces au sein de cette famille que dépeignent les romanciers haïtiens. C'est dans le récit de Justin Lhérisson, La Famille des Pitite-Caille. On a toujours souligné l'originalité de ce récit que son auteur a voulu qualifier d'audience et non pas de roman pour bien montrer sa fidélité à une forme de la tradition orale populaire dont il s'inspirait. Mais on n'a pas encore relevé que ce récit plein d'humour et d'esprit était aussi un roman d'anticipation. Qu'arriverait-il, en effet, si une sœur qui veut se porter à la rescousse de son frère, non pas un peu trop tard, comme Mama, ou pas du tout, comme Cilotte Thazar, s'était décidée plutôt à lui offrir le bonheur clés en mains, comme on dit, dès le départ ? Ce serait un véritable conte de fées. Et pourtant l'audience de Justin Lhérisson nous fait voir que rien n'est moins sûr car il y aurait une dernière barrière à surmonter, la plus haute sans doute : celle de s'assurer de ce que le bénéficiaire ferait de ce cadeau.

Le titre même de cette audience : La famille des Pitite-Caille, (1905), ce qui pourrait se traduire par "La famille des enfants de la maison », faisait d'emblée de ce récit une métaphore individualisée d'une situation collective passée que l'on avait la chance de transformer en situation moderne. Mais pour cela il aurait fallu que le bénéficiaire du cadeau que lui fait sa femme eût une vision du passé à dépasser et du futur à bâtir. Avoir en quelque sorte un plan pour aujourd'hui et un pour demain.

Le héros du récit de Lhérisson, Eliézer Pitite-Caille, le seul fils qu'eût son père de sa femme légitime, est sans nul doute le plus chanceux des enfants d'une famille par ailleurs fort étendue. En effet, après avoir roulé sa bosse et pratiqué tous les métiers jusqu’à finalement devenir marin, Eliézer eut le bonheur de rencontrer une Martiniquaise, Velléda, une "tireuse de carte " (voyante) dont la clientèle était si nombreuse qu'elle était devenue riche. 
Eliézer l'épousa et grâce à la dot inattendue que lui apportait sa femme, commença par faire, en guise de lune de miel, un agréable voyage à Paris. Ensuite le couple vint s'installer dans un quartier chic de la capitale.

Arrêtons-nous, un instant, sur le profil de cette épouse d'Eliézer. Martiniquaise, donc antillaise d'ascendance mi-africaine mi-française, Velléda nous fait immédiatement penser à ces deux personnages de nationalité française qui ont joué un même rôle d'adjuvante qu'elle. Il s'agit d'une part, de la française, Stella, qui, dans le roman d'Emeric Bergeaud, avait aidé les jumeaux Romulus et Rémus dans leur lutte pour l'indépendance d'Haïti. Et, d'autre part, de la journaliste étrangère, française selon toute probabilité, qui était devenue la muse de l'étudiant dans le roman Bicentenaire de Lyonel Trouillot. Avec cette différence que si ces deux adjuvantes sont définitivement retournées en France, Velléda, elle, après un bref crochet par Paris, pour sa lune de miel, est revenue vivre à Port-au-Prince avec son mari. L'adjuvante qui soutenait le protagoniste est donc revenue vivre avec lui, sur les lieux mêmes de leur combat. Avec la dot de sa femme, Eliézer qui jusque là tirait le diable par la queue, aurait pu mener une vie paisible, ou mieux encore, voir à faire fructifier son capital.

Mais au lieu de se contenter d'une vie pacifique, Eliézer, pris d'une sorte de folie des grandeurs, commença par se faire membre d'une loge franc-maçonnique pour ensuite entreprendre de se faire élire comme député du peuple. Mal lui en prit, les autorités en place s'empressèrent de freiner cette ascension à laquelle il aspirait. Il fut donc arrêté, jeté en prison et n’en sortira que pour mourir. Entretemps il avait été ruiné et ses deux enfants avaient connu un sort tout aussi lamentable que le sien. Quant à sa femme, au bout de tous ces déboires, elle finira par devenir la maîtresse d'un de ces potentats qu'Eliézer Pitite-Caille avait eu la mauvaise idée d'affronter.

Avec une telle intrigue, Justin Lhérisson écrivait un récit d'anticipation plutôt qu'une simple relation de faits présents ou passés. En effet la vengeance de Mama s'était terminée par son suicide et les épousailles de Cilotte Thazar ne lui avait pas rapporté le bonheur qu’elle souhaitait. Or le résultat n'est guère meilleur pour Eliézer Pitite-Caille qui avait pourtant bien commencé sa vie familiale. La réponse à la question implicite de Lhérisson, c’est Jacques Roumain qui viendra, dans Gouverneurs de la rosée, commencer à nous la dévoiler.

Gouverneurs de la rosée

Cette mise en évidence du rôle d'adjuvant que joue la sœur et de la variété des positions qu'elle pouvait prendre dans les œuvres des romanciers 
de La Ronde permet de saisir le tournant que va prendre Gouverneurs de la rosée de Jacques Roumain en montrant une sœur qui poursuit la lutte du frère en s'associant avec la mère de celui-ci. Voilà qui est l'inverse du comportement de Cilotte Thazar qui s'allie avec sa mère pour repousser le prétendant qu'elle préférait.

Dans Gouverneurs de la rosée qui parut en 1944, Délira, la mère de Manuel, après la mort de celui-ci, réussit à sauver in extremis, le projet que présentait son fils à ses voisins de Fonds-rouge, pour la sauvegarde de leur village. La communauté de Fonds-Rouge était divisée en deux clans ennemis. Manuel et Gervilen étaient les porte-parole de ces deux groupes. Quand Manuel viendra proposer la paix à ses ennemis, Gervilen l'assassinera. Mais l'intervention de Délira, la mère du défunt, secondée par Annaise, membre du clan de Gervilen mais qui avait rejoint celui de Manuel, permettra de sauver le projet de réconcilier les habitants du village

Cette intervention de la mère est appuyée par une sœur. Annaïse en effet se déclare-fille de Delira en l'appelant : "Maman ». Par cette parole, elle devient en quelque sorte la sœur de son défunt amoureux. L'association positive de la mère et de la sœur, dans Gouverneurs de la rosée, renversait l'alliance négative de Madame Thazar et de sa fille, Cilotte, puisque cette fois-ci, cette alliance conduisait l'histoire à une fin sinon heureuse du moins satisfaisante.

Cette alliance d'une mère et d'une sœur, comblait le vide que l'on pouvait constater dans les romans de Bergeaud et de Trouillot, par l'absence des mères, Marie l'Africaine et Ernestine Saint-Hilaire, et le départ des sœurs qu'étaient l'Ange de la liberté et la journaliste étrangère. En même temps elle préparait le passage de la sœur de la fonction d'adjuvante à celle de protagoniste de l'action. Le succès de la démarche conjointe de Delira et d'Annaïse le laisse présager et cela se confirmera dans les romans de Marie Chauvet.

\section{Amour et Colère de Marie Chauvet}

Si l'intervention de Delira assistée par Annaïse a assuré la survie du plan de Manuel, elle n'a pas pu sauver la vie de celui-ci puisqu'il était déjà mort quand les deux femmes sont intervenues. C'est cette intervention heureuse et surtout en temps opportun que vont réussir à faire Claire et Rose, dans les deux premiers récits de la trilogie de Marie Chauvet : Amour, Colère et Folie publiée en 1968.

L'histoire de Claire, dans Amour, est complexe. Elle a pris la direction de la maison familiale et cohabite avec ses deux sœurs. L'une est célibataire et l'autre est mariée à un Français, Jean Luze. Le couple a une fille dont Claire est la marraine. Tout cela fait de Claire un véritable chef de famille. Or Claire 
aime en secret Jean-Luze, son beau-frère, avec qui elle partage d'ailleurs les mêmes convictions politiques progressistes. Elle ne pourrait donc pas se comporter en rivale de sa sœur, donc en donnant libre cours à son amour, sans plonger sa famille dans le malheur. Mais en même temps Claire est courtisée par Calédu, le tonton macoute qui règne en maître sur la ville. Elle ne pouvait pas s'associer à ce tyran, sans renoncer à ses convictions politiques et surtout sans contribuer à augmenter le malheur de ses concitoyens. Double dilemme donc, qu'elle dénoue par un double sacrifice. Après une longue et douloureuse introspection, elle choisit de renoncer à son amour pour son beau-frère et de tuer le tonton-macoute qui terrorisait la ville. On peut parler de double sacrifice puisqu'il s'agit d'une victoire remportée au prix d'un double renoncement personnel. Et peut-être même pourrait-on dire pour une double satisfaction. L'appui au bonheur de sa famille est aussi la satisfaction d'appuyer un beau-frère dont elle partage les convictions politiques. La mort de Calédu est une satisfaction pour la ville qui est délivrée d'un tyran et pour Claire d'être débarrassée d'un prétendant indésirable.

Or on aura remarqué que Claire parvient à ce dénouement en assumant, seule, la double fonction de protagoniste et d'adjuvant que remplissaient séparément Delira et Annaïse. Elle se fait héautontimoroumenos, bourreau de soi-même, par deux fois, au profit de deux groupes de bénéficiaires : sa famille et sa communauté. Ainsi peut-elle assurer une fin heureuse au combat dans lequel elle était plongée.

Dans Colère, le second récit de la trilogie de Marie Chauvet, l'histoire est peut-être moins complexe mais pas moins cruelle. Rose, accepte délibérément de devenir l'objet, la victime des fantasmes sexuels du tonton-macoute qui la convoitait. Mais c'est grâce à ce sacrifice qu'elle permet à son frère d'obtenir la permission de quitter le pays. Elle sauve la vie de son frère en sacrifiant la sienne. Autrement dit en remplissant la double fonction d'un protagoniste acceptant d'être l'objet d'un ennemi commun. Un résultat qu'expliquait déjà le titre du dernier chapitre de Gouverneurs de la rosée : "La fin et le commencement.»

En effet tout dénouement positif se caractérise par une fin qui est en même temps un commencement. On ne met fin à une situation malheureuse que pour commencer une nouvelle plus heureuse. De ce point de vue on peut comprendre par exemple l'échec d'Eliézer Pitite-Caille qui voulait bien changer sa condition personnelle mais sans changer la condition collective de la population qu'il voulait représenter comme député. Cela vaut aussi pour Marilisse qui n'avait pas d'autre raison de vivre que celle de se venger. Et tout autant pour Cilotte Thazar, à qui sa trahison à elle-même et à son prétendant n'apporte pas la vie heureuse qu'elle espérait. Autrement dit le bonheur que l'on 
veut avoir plus tard exige des sacrifices dans le moment présent. Et Manuel en était bien conscient puisque c'est sur sa recommandation que sa mère et Annaïse ne dénonceront pas celui qui l'a tué. Il acceptait de mourir si cela devait contribuer à faire triompher la cause qu'il défendait. C'est ce même genre de sacrifice auquel les deux héroïnes d'Amour et de Colère consentent.

Autrement dit, il faut avoir une vision de la situation présente à changer et du renouveau qu'apporterait la situation qui allait remplacer la première. Avoir en même temps un plan pour aujourd'hui et un autre pour demain et surtout consentir d'avance au sacrifice qu'exigera ce passage. Or ce sacrifice se mesure à l'aune de la mort que Manuel et Rose acceptent par avance.

La lutte des femmes haïtiennes

Dès le début de la Révolution haïtienne, en 1802, les femmes ont soutenu, les armes à la main, la lutte de libération nationale comme l'illustrent les noms de femmes célèbres, comme Sanite Bélair, Marie-Jeanne Lamartinière ou Catherine Flon. Mais les femmes, pour la plupart et de tout temps, ont mené la lutte en étant la base arrière qui soutenait ceux qui se battaient sur le front. Les figures romanesques de Marie l'Africaine, dans Stella, ou d'Ernestine St Hilaire, dans Bicentenaire, représentent cette armée souffrante de l'arrière-garde.

De 1804 à 1915, les Haïtiens semblent n'avoir eu qu'une seule obsession : celle de mettre en place un gouvernement politiquement correct, selon les normes européennes. De là cette persistance à ne pas faire autre chose que promulguer de nouvelles constitutions à chaque prise du pouvoir, à la suite d'une insurrection militaire. Il aura fallu Gouverneurs de la rosée de Jacques Roumain pour proposer désormais comme objectifs d'une nouvelle poétique indigéniste, non pas seulement l'utilisation de la langue créole ou l'éloge de la culture populaire mais la volonté chez les personnages romanesques de travailler à assurer le bien-être collectif de leurs concitoyens comme c'est le cas de Manuel pour les habitants de Fonds-Rouge.

C'est dans cette même veine que s'inscrit la création de la première tragédie écrite en langue créole, l'Antigòn (1963) de Félix Morisseau-Leroy qui est une adaptation de la pièce de Sophocle représentant, comme on le sait, la révolte d'une sœur qui défendait la mémoire d'un frère mort à qui elle voulait donner une sépulture honorable. Or si on réexamine cette imitation de Sophocle par Morisseau-Leroy, on doit le faire aussi pour une chanson comme : "Ti manman pa kite $\mathrm{m}$ ale " ou pour cette autre chanson où celui qui parle souhaite mourir dans les bras de la mère Haïti. On verra qu'il s'agit de la même supplique d'un frère-fils qui s'adresse à une sœur-mère pour qu'elle le maintienne en vie ou le fasse renaître. 
S'il fallait résumer l'histoire que nous racontent les romanciers haïtiens, depuis la publication de leur premier roman, je dirais que c'est l'histoire d'une lutte que mènent deux frères qui d'abord combattirent ensemble contre un ennemi étranger. Ils furent aidés dans cette lutte par des sœurs venues de l'étranger. Mais une fois cette lutte terminée, les frères se sont lancés dans une nouvelle guerre, cette fois entre eux-mêmes. Ces premières sœurs se sont alors retirées pour céder la place à d'autres sœurs, plus proches des combattants. Elles étaient chargées non pas d'appuyer la lutte fratricide des frères mais de les réconcilier. Ces sœurs échouèrent d'abord dans cette mission. Soit en s'y prenant trop tard, soit par mauvaise foi soit par l'absence de collaboration de la part des frères. Mais quand mère et sœur joignirent leurs efforts, de protagoniste et d'adjuvant, les deux purent sauver l'héritage d'un frère mort au combat et assurer ainsi une première forme de réconciliation. Cela ne mit pas fin pour autant à la querelle des frères. Mais la sœur agissant désormais dans la double fonction de protagoniste et d'adjuvante est parvenue, à force de sacrifices, à éviter au moins la mort du frère de sorte que l'avenir demeure ouvert.

Il en résulte que " a luta continua». Cela est d'autant plus vrai, qu'un roman relativement récent, puisqu'il fut publié après les récits de Marie Chauvet, vient de démontrer qu'il reste au frère à entrer dans le jeu pour appuyer la sœur dans sa démarche de réconciliation.

En 1975, Frankétienne a publié Dezafi, le premier roman écrit en langue haïtienne. Ce récit nous raconte une histoire encore plus troublante que celle d'Eliézer Pitite-Caille. Si ce dernier avait déçu par son comportement après avoir reçu l'aide de sa femme Velleda, le comportement de Klodonis, dans Dezafi, est plus troublant et nous laisse même perplexe.

Résumons les faits qui sont racontés dans Dezafi. Klodonis, jeune activiste militant contre les injustices sociales, est réduit à l'état de zombi par Zofè, un oungan (sorcier). Mais Siltana, la fille du oungan, s'éprend de Klodonis et le délivre de son état de zombi en lui donnant du sel à avaler.

Rappelons que, selon le mythe de la zombification, donner du sel à goûter à un zombi le tire de sa léthargie et lui permet de reprendre une pleine conscience de sa situation. Ajoutons que si Emeric Bergeaud, au début de l'histoire du roman haïtien de langue française s'était servi du mythe de la fondation de Rome par les jumeaux Romulus et Rémus, Frankétienne reprend le même procédé. Il se sert pour écrire le premier roman en langue créole d'un mythe du vodoun, celui de la zombification. Mythe qui n'est pas sans rapport avec cet autre mythe du vodoun qui veut que les jumeaux marassas soient tout-puissants quand ils s'entendent et sans force quand ils ne s'en- 
tendent pas. Toute lutte entre frères ennemis se ramènerait, en somme, à la volonté de chacun de zombifier l'autre.

Frankétienne procède à un réamenagement du schéma traditionnel de la lutte des frères ennemis. En faisant de Sintil un père incestueux de sa fille Siltana, il en fait un personnage double, à la fois père et frère de cœur de sa fille. Voilà qui le place dans la fonction de rival de Klodonis puisque Siltana se déclare ouvertement amoureuse de Klodonis. Par cette déclaration, elle donnait la priorité à celui qu' elle voyait comme son frère face à ce père à deux visages. Or quand Siltana, par amour pour Klodonis, lui donne du sel à goûter et que ce dernier sort de son hébétude, la première réaction de ce mort redevenu vivant est de donner une gifle épouvantablement forte à sa libératrice : «Li koupe Siltana youn patasouèl krazebrizédémantibiléblayividé " (FRANKÉTIENNE, 1975: 295) (Il lui flanqua une gifle-à écraser-briser-détruire-jeter par terre-à couper le souffle). La seule enfilade de ces épithètes agglutinées suffit à faire comprendre la force et la férocité de cette réaction étonnante de quelqu'un qui bénéficiait pourtant d'un geste fait par amour et pour sa délivrance.

La seule remarque que l'on puisse faire alors c'est de constater que l'inconscience d'Eliézer Pitite-Caile gaspillant, en 1905, la chance que lui donnait Velléda, n'avait pas cessé en 1975 . Tout comme l'imprudence d'Eliézer ruinait tout l'effort fait par Velléda, l'ingratitude ou l'aveuglement de Klodonis n'augurait rien de bon pour l'avenir. Force est de conclure que si dans cette lutte commune la sœur avait amplement fait sa part, il reste encore au frère à faire la sienne.

Un espoir demeure cependant. On remarquera que les nouvelles héroïnes arrivent à prendre l'attitude qu'elles adoptent à la suite d'un long et sincère examen de conscience où elles mettent leur âme à nu. C'est ce qu'illustre fort bien le cas de Claire, dans Amour. Le récit, dans ce roman, n'est rien d'autre que la lecture que fait Claire du journal qu'elle tient. Un signe sans doute que nous fait Marie Chauvet pour suggérer que ses personnages, notamment les frères ennemis, et nous-mêmes, lecteurs, nous devrions en faire autant. C'està-dire : rentrer dans notre âme et conscience et nous regarder sans fard. En fait cette prise de conscience avait commencé déjà dans Gouverneurs de la rosée mais l'art d'écrire de Marie Chauvet rend plus sensible cet exercice pour le lecteur.

On ne peut pas donner de réponse à une question qu'on ne s'est jamais posée. Ce sont les faits qui nous amènent à nous interroger. C'est pour cela qu'on peut dire : "a luta continua ». La lutte des femmes et des hommes se poursuit comme une histoire qui doit finir pour recommencer autrement. Avant Roumain, ce qui veut dire avant l'occupation états-unienne de 1915 et la révolution bolchévique de 1917, à l'époque de La Ronde, en somme, 
on ne voyait pas les choses comme on les voit depuis. Il en va de même pour Marie Chauvet qui ne devait pas s'attendre à voir les zinglins de Soulouque et les fiyet lalo de Salnave renaitre sous le nom de tonton macoute pendant le règne de François Duvalier. On définit généralement la tradition comme une mémoire vivante de la collectivité. Si tel est le cas, on doit admettre que ce n'est que depuis peu que les Haïtiens se souviennent de leur passé par écrit. D'abord ce fut en français et plus récemment en haïtien. Par ailleurs les traditions peuvent, dans les pays de vieille civilisation, remonter à des millénaires. Mais pour les sociétés de formation récente, elles prennent du temps à se faire ou à se défaire. Alors il faut, comme nous y invite Antonio Candido, distinguer, dans la masse des récits, les " manifestations littéraires » de la " littérature " proprement dite, laquelle doit être perçue comme un système. Ce qui permet de distinguer les phases de l'évolution de ce système.

Comme on le voit, par les différentes exigences que fait le critique brésilien, l'analyse d'une tradition est pour le moins compliquée dans un pays comme Haïti où plusieurs des divers pré-requis énumérés dans Formação da literatura brasileira tardent à se matérialiser dans la réalité. Songeons d'abord à l'analphabétisme, barrière tant à la constitution d'un public de lecteurs qu'à la floraison de maisons d'éditions. Et comme cet analphabétisme vaut autant pour la connaissance de la langue française que de l'haïtienne et qu'il se double de la précarité des moyens dont disposent les éventuels lecteurs, on comprendra que cela constitue autant de facteurs susceptibles de freiner aussi bien la constitution du public lecteur que la prolifération des créateurs de la plume. De là, notamment cette obligation de se référer à l'oral pour expliquer ce qui est écrit

Il y aurait d'ailleurs bien d'autres handicaps à relever. Néanmoins les conditions posées par l'auteur de Formação da literatura brasileira nous permettent de faire un état de la question et de mesurer la distance parcourue dans la formation de la littérature haïtienne aussi bien de langue française qu'haïtienne.

$\mathrm{Si}$, comme le dit Antonio Candido, la tradition littéraire est un système symbolique qui permet à un individu de communiquer au reste du monde sa vision et son interprétation de la réalité, on peut dire qu'Emeric Bergeaud focalise l'attention sur la mère mise à mort. Lyonel Trouillot le fait sur la mère, infirme et abandonnée à son sort. Jacques Roumain par contre nous montre la mère et la sœur se dressant pour sauver la croisade du fils et frère tandis que Marie Chauvet et Frankétienne nous présentent la sœur qui vole au secours de son frère et, pour la première fois, le libère.

Chacun de ces écrivains, à sa manière et selon son époque, s’appuient sur cette focalisation de la vision haïtienne sur la femme pour nous montrer que 
l'héroïsme n'est point tant une question de sexe qu'une capacité de faire un examen de conscience en toute honnêteté. Amour de Marie Chauvet, disionsnous, en est un exemple par ce dévoilement d'elle-même que fait Claire à la lecture de son propre journal. Cela lui permet de se voir sans fard, comme dans un miroir, et de tirer, en toute sincérité, les conclusions auxquelles l'amènent ses propres pensées sur sa vie aussi bien individuelle que sociale.

\section{Références}

BERGEAUD, Emeric. Stella. Paris : Dentu, 1859.

BERROU, F.Raphël; POMPILUS, Pradel. Histoire de la littérature haïtienne illustrée par les textes. T. 11, Port-au-Prince : Éditions Caraïbes; Paris : Éditions de l'École, 1975.

CANDIDO, Antonio. Formação da literatura brasileira. vol.1, 7a edição, Belo Horizonte : Rio de Janeiro, Itatiaia, 1993.

CHAUVET, Marie. Amour, Colère et Folie. Paris: Gallimard, 1968.

FRANKÉTIENNE. Dézafi, Port-au-Prince,: Édision Fardin, 1975.

HIBBERT, Fernand. Les Thazar. Port-au-Prince: Éditions Henri Deschamps, 1988. Collection Esquisses d'Hier, Tableaux d'Aujourd'hui.

LHÉRISSON, Justin. Les oeuvres romanesques de Justin Lhérisson: La Famille des PititeCaille et Zoune chez sa ninnaine. Paris : Éditions Caribéennes, 1978.

MORISSEAU-LEROY, Félix.( Moriso-Lewa) Teyat kreyòl. Delmas ( Port-au-Prince) : Editions Libète, 1997

ROUMAIN, Jacques. Euvres complètes, édition critique de Léon-François Hoffmann. Paris : ALLCA XX, 2003. Collection Archivos.

TROUILLOT, Lyonel. Bicentenaire. Arles /Montréal : Actes Sud/ Leméac, 2004.

Maximilien Laroche é Professor Titular da Université Laval (Québec, Canadá). Doutor pela Université de Toulouse. Autor de Le miracle et la métamorphose (1970), L'image comme écho (1978), Littérature haïtienne, identité, langue, réalité (1981), L'avènement de la littérature haïtienne (1987), La double scène de la représentation (1991), Dialectique de l'américanisation (1993), La Sémiologie des apparences (1994), Mythologie haïtienne (2002), Littérature Haïtienne comparée (2007).

E-mail: Maximilien.Laroche@sympatico.ca 